\title{
Rab1 GTPase and Dimerization in the Cell Surface Expression of Angiotensin II Type 2 Receptor
}

\author{
Xiaoping Zhang, Guansong Wang, Denis J. Dupré, Yumei Feng, Mélanie Robitaille, \\ Eric Lazartigues, Ying-Hong Feng, Terence E. Hébert, and Guangyu Wu
}

Department of Pharmacology and Experimental Therapeutics, Louisiana State University Health Sciences Center, New Orleans, Louisiana (X.Z., G.Wa., Y.F., E.L., G.Wu.); Department of Pharmacology and Therapeutics, McGill University, Montreal, Quebec, Canada (D.J.D., M.R., T.E.H.); and Department of Pharmacology, Uniformed Services University of the Health Sciences, Bethesda, Maryland (Y.H.F.)

Received March 11, 2009; accepted April 7, 2009

\begin{abstract}
The physiological function of angiotensin II (Ang II) is mediated through the Ang II type 1 (AT1R) and type 2 (AT2R) receptors. Our previous studies have demonstrated that cell surface targeting of AT1R is regulated by Rab and Sar1 GTPases and the $\mathrm{F}(\mathrm{x})_{6} \mathrm{LL}$ motif in the membrane-proximal $\mathrm{C}$ terminus. However, the molecular mechanisms underlying the export of nascent AT2R remain poorly defined. In this report, we determined the role of Rab1 GTPase, which specifically controls protein transport from the endoplasmic reticulum (ER) to the Golgi, and receptor dimerization in the biosynthesis of AT2R. Cell surface expression of AT2R was augmented by transient expression of Rab1 and attenuated by dominant-negative Rab1 mutants and small interfering RNA-mediated knockdown of Rab1. Consistently, AT2R inhibition of epidermal growth factor-activated extracellular signal-regulated kinase 1/2 was significantly re-
\end{abstract}

duced by the Rab1 mutants, indicating that endogenous Rab1 modulates the cell surface targeting and signaling of AT2R. It is of interest to note that Rab1 augmented the overall expression of AT2R and its mRNA, whereas the Rab1 mutants attenuated the total AT2R expression and enhanced ubiquitin-dependent AT2R degradation. Furthermore, our previously characterized ER export-deficient AT1R mutant in which the $\mathrm{F}(\mathrm{x})_{6} \mathrm{LL}$ motif was mutated formed both homodimers and heterodimers with AT2R. Dimerization of the AT1R mutant with AT2R blocked AT2R trafficking to the cell surface, suggesting constitutive dimerization of both receptors in the ER and an important role of dimerization in ER export of the receptors. These data demonstrate for the first time that Rab1 GTPase and dimerization modulate export traffic from the ER to the cell surface of newly synthesized AT2R.
Angiotensin II (Ang II) plays an important role in modulation of the physiological function of virtually all organs and in the development of many diseases, such as diabetes, hypertension, myocardial infarction, congestive heart failure, and stroke (Murphy et al., 1991; Hein et al., 1995; Gallinat et al., 2000; Hunyady and Catt, 2006; Mehta and Griendling, 2007). The function of Ang II is mediated through activation of the cell surface Ang II type 1 receptor (AT1R) and Ang II type 2 receptor (AT2R), both of them belong to the seven transmembrane-spanning receptor superfamily coupled to

This work was supported by the National Institutes of Health National Institute of General Medical Sciences [Grants GM76167, HL64592]; and the Canadian Institutes of Health Research.

Article, publication date, and citation information can be found at http://jpet.aspetjournals.org.

doi:10.1124/jpet.109.153460 heterotrimeric G proteins (GPCRs). It has been well demonstrated that AT1R mainly couples to the $G_{q}$ family $G$ proteins to activate several downstream effectors, whereas the function of AT2R is largely mediated through $\mathrm{G}_{\mathrm{i}}$ to antagonize the AT1R action (Kang et al., 1994; Huang et al., 1996; Zhang and Pratt, 1996; Matsubara, 1998; Hunyady and Catt, 2006).

Similar to signaling mechanisms, the intracellular trafficking of AT1R and its role in regulating receptor function and in the pathogenesis of certain diseases have been extensively studied (Matsubara, 1998; Mehta and Griendling, 2007). These studies have strongly demonstrated that the function of AT1R is critically regulated by its intracellular trafficking, including endocytosis, recycling, degradation, and maturation. We have recently demonstrated that AT1R exit from the endoplasmic reticulum (ER) is mediated by a motif consisting of a phenylalanine and a dileucine spaced by six residues

ABBREVIATIONS: Ang II, angiotensin II; AT1R, angiotensin II type 1 receptor; AT2R, angiotensin II type 2 receptor; GPCR, G protein-coupled receptor; ER, endoplasmic reticulum; HEK, human embryonic kidney; AT1Rm, angiotensin II type 1 receptor mutant; HA, hemagglutinin; ERK, extracellular signal-regulated kinase; GFP, green fluorescent protein; EGF, epidermal growth factor; PCR, polymerase chain reaction; PBS, phosphate-buffered saline; FBS, fetal bovine serum; siRNA, small interfering RNA; PAGE, polyacrylamide gel electrophoresis; MG132, $N$ benzyloxycarbonyl (Z)-Leu-Leu-leucinal; AR, adrenergic receptor; ALK, activin-like kinase. 
$\left[\mathrm{F}(\mathrm{x})_{6} \mathrm{LL}\right]$ in the membrane-proximal $\mathrm{C}$ terminus and a single leucine residue in the first intracellular loop of the receptor (Duvernay et al., 2004, 2009; Zhou et al., 2006). We have also demonstrated that the cell surface expression of AT1R and AT1R-mediated signaling in HEK293 cells and in cardiomyocytes can be manipulated through modifying the function of the Ras-like small GTPases, including Rab1, Rab2, Rab6, and Sar1 (Wu et al., 2003; Filipeanu et al., 2004, 2006; Dong and Wu, 2007; Dong et al., 2008), which are involved in protein transport along the early secretory pathway. Rab1 specifically regulates anterograde protein transport from the ER to the Golgi, and Sar1 coordinates the formation of ERderived COPII-coated vesicles on the ER membrane that exclusively mediate protein exit from the ER, whereas Rab2 and Rab6 are involved in the retrograde protein transport between the ER and Golgi (Plutner et al., 1991; Jin et al., 1996; Lupashin and Waters, 1997; Allan et al., 2000; Sato and Nakano, 2004; Stephens and Pepperkok, 2004; AltanBonnet et al., 2006). In contrast to AT1R, the molecular mechanisms underlying the intracellular trafficking, particularly export from the ER through the Golgi to the cell surface of AT2R, remain largely unknown.

Dimerization has been well described for a variety of GPCRs. Whereas some GPCRs constitutively dimerize in the $\mathrm{ER}$ and are then transported to the cell surface, other receptors may form dimers at the plasma membrane in response to agonist stimulation (Jones et al., 1998; Overton and Blumer, 2000, 2002; Szidonya et al., 2008). Although the precise function of receptor dimerization in vivo needs further investigation, many studies have demonstrated that GPCR dimerization may play an important role in regulating receptor folding, maturation, intracellular trafficking, ligand binding selectivity, and signaling. Similar to many other GPCRs, both AT1R and AT2R have been demonstrated to form homoand heterodimers (AbdAlla et al., 2004; Hansen et al., 2004; Miura et al., 2006). In this report, as an initial approach to elucidating the molecular mechanism of export trafficking of newly synthesized AT2R, we determined the role of Rab1 GTPase and receptor dimerization in the assembly, export, and signal regulation of functional AT2R. Our data indicate that the ER-to-Golgi movement of nascent AT2R is modulated by Rab1 GTPase and that dimerization of AT2R occurs constitutively in the ER and controls ER export of the receptor. These data also suggest that AT2R signaling could be manipulated by modifying its export trafficking along the secretory pathway.

\section{Materials and Methods}

Materials. Angiotensin II type 1A receptor in vector pCDM8 and hemagglutinin (HA)-tagged ubiquitin (Ballabeni et al., 2004) were kindly provided by Dr. Kenneth E. Bernstein (Department of Pathology, Emory University, Atlanta, GA) and Dr. Ashok D. Pullikuth (Department of Pharmacology, Louisiana State University Health Sciences Center, New Orleans, LA). Antibodies against Rab1, Rab2, Rab4, Rab6, phospho-ERK1/2, and green fluorescent protein (GFP); rhodamine-conjugated anti-HA antibody; and protein G PLUS-agarose beads were purchased from Santa Cruz Biotechnology, Inc. (Santa Cruz, CA). High-affinity fluorescein-conjugated anti-HA antibody 3F10 was from Roche Applied Science (Mannheim, Germany). Human Ang II and EGF were purchased form Calbiochem (San Diego, CA). Penicillin-streptomycin and L-glutamine were from Invitrogen (Carlsbad, CA). Alexa Fluor 594-labeled secondary antibod- ies and 4,6-diamidino-2-phenylindole were from Invitrogen. All other materials were obtained as described previously (Wu et al., 2001; Dong and $\mathrm{Wu}, 2007$ ).

Plasmid Constructions. To generate the pcDNA3.1(-) vector containing $3 \mathrm{HA}$ at the $\mathrm{XbaI}$ and $\mathrm{XhoI}$ restriction sites, two primers (forward primer, 5'-CTAGAATGTACCCATACGATGTTCCAGATTACGCTTACCCATACGATGTTCCAGATTACGCTTACCCATACGATGTTCCAGATTACGCTGATC-3'; and reverse primer, 5'-TCGAGATCAGCGTAATCTGGAACATCGTATGGGTAAGCGTAATCTGGAACATCGTATGGGTAAGCGTAATCTGGAACATCGTATGGGTACATT-3') encoding three YPYDVPDYA and containing XbaI and XhoI restriction sites were synthesized, annealed, and ligated into the pcDNA3.1(-) vector (Invitrogen), which was digested with $\mathrm{XbaI}$ and XhoI. To generate AT2R tagged with $3 \mathrm{HA}$ at its $\mathrm{N}$ terminus, the full-length AT2R was amplified by polymerase chain reaction (PCR) using the forward primer 5'-GCGCCTCGAGATGAAGGACAACTTCAGTTTTG-3' and the reverse primer 5'-GCATAAGCTTTTAAGACACAAAGGTGTCCATTTC-3'. The PCR product was digested with XhoI and HindIII, purified, and ligated to HA-tagged pcDNA3.1(-) vector, which was digested with XhoI and HindIII. AT2R conjugated with GFP at its C terminus and tagged with Myc or FLAG at its N terminus was generated as described previously (Feng et al., 2002). These epitopes have been used to label GPCRs, resulting in the receptors with similar characteristics to the wild-type receptors. The ER export-defective AT1R mutant (AT1Rm) in which the $F(X)_{6} L L$ motif in the AT1R C terminus was mutated to alanines was generated as described previously (Duvernay et al., 2004). To construct plasmids coding for human AT1R (wild type and mutant) and AT2R with an N- or C-terminal fragment of the GFP variant Venus, the receptors were amplified by PCR using the following primers: AT1R venus (Not1) forward primer, 5'-CGGGGCGGCCGCACCATGGCCCTTAACTCTTCT-3'; AT1R venus (Cla1) reverse primer, 5' GCCACCATCGATCTCCACCTCAAAACAAGACGC-3'; AT2R venus (Not1) forward primer, 5'-CGGGGCGGCCGCACCATGAAGGACAACTTCAGTTTTGCT-3'; and AT2R venus (Cla1) reverse primer, 5'-GCCACCATCGATAGACACAAAGGTGTCCATTTC-3'. The resulting fragments were cloned to replace the GCN4 leucine zipper from pcDNA3.1/Zeo(+)-GCN4 leucine zipper-Venus1 and pcDNA3.1/ Zeo(+)-GCN4 leucine zipper-Venus2 cDNAs. Rab1 GTPase was tagged with the FLAG epitope at its N terminus (FLAG-Rab1) as described previously (Wu et al., 2003). Rab1 dominant-negative mutants (S25N, a GDP-bound form and N124I, a guanine nucleotide binding-deficient form) were generated using QuikChange sitedirected mutagenesis (Stratagene, La Jolla, CA). These Rab1 mutants have been well characterized and extensively used to explore the function of endogenous Rab1 (Wu et al., 2003). The structure of each construct used in the present study was verified by restriction mapping and nucleotide sequence analysis.

Cell Culture and Transient Transfection. HEK293 cells were cultured in Dulbecco's modified Eagle's medium with $10 \%$ fetal bovine serum, 100 units/ml penicillin, and $100 \mathrm{units} / \mathrm{ml}$ streptomycin and transiently transfected by using Lipofectamine 2000 reagent (Invitrogen) as described previously (Wu et al., 2003). Transfection efficiency for both 35- and 100-mm dish formats was estimated to be greater than $70 \%$ based on the measurement of GFP fluorescence.

Flow Cytometric Analysis of Receptor Expression. For measurement of total AT2R expression, HEK293 cells transfected with AT2R-GFP were collected and resuspended in PBS containing 1\% FBS at a density of $8 \times 10^{6}$ cells $/ \mathrm{ml}$. The overall receptor expression was determined by measuring total GFP fluorescence on a flow cytometer (FACSCalibur; BD Biosciences, San Jose, CA) as described previously (Wu et al., 2003). For measurement of AT2R expression at the cell surface, HEK293 cells were cultured on sixwell plates and transfected with HA-AT2R together with Rab1 mutants for $48 \mathrm{~h}$. The transfected cells were collected, suspended in PBS containing $1 \% \mathrm{FBS}$ at a density of $8 \times 10^{6} \mathrm{cells} / \mathrm{ml}$, and incubated with high-affinity anti-HA-fluorescein $(3 \mathrm{~F} 10)$ at a final concentration of $2 \mu \mathrm{g} / \mathrm{ml}$ for $30 \mathrm{~min}$ at $4^{\circ} \mathrm{C}$. After washing three times with $1.0 \mathrm{ml}$ 
of PBS/1\% FBS, the cells were resuspended and the fluorescence was analyzed as described above. Because the staining with the anti-HA antibodies was carried out in the unpermeabilized cells and only those receptors expressed at the cell surface were accessible to the anti-HA antibodies, the measured fluorescence reflected the amount of receptor expressed at the cell surface.

Fluorescence Microscopy. For fluorescence microscopic analysis of receptor subcellular localization, HEK293 cells were grown on coverslips precoated with poly-L-lysine in six-well plates and transfected with $500 \mathrm{ng}$ of GFP-tagged receptor cDNA. The cells were fixed with ice-cold methanol $\left(-20^{\circ} \mathrm{C}\right)$ for $10 \mathrm{~min}$ and stained with 4,6-diamidino-2-phenylindole for $5 \mathrm{~min}$. For subcellular localization of AT2R tagged with HA or Myc, HEK293 cells were permeabilized with PBS containing $0.2 \%$ Triton X-100 for 5 min and blocked with $5 \%$ normal donkey serum for $2 \mathrm{~h}$. The cells were then incubated with antibodies against HA or Myc at a dilution of 1:50 for $1 \mathrm{~h}$. After washing with PBS ( $3 \times 5$ min), the cells were incubated with Alexa Fluor 594-labeled secondary antibody (1:2000 dilution) for $1 \mathrm{~h}$ at room temperature. The coverslips were mounted, and fluorescence was detected with a DMRA2 epifluorescent microscope (Leica Microsystems, Inc., Deerfield, IL) as described previously (Wu et al., 2003). Images were deconvolved using SlideBook software and the nearest neighbor deconvolution algorithm (Intelligent Imaging Innovations, Denver, CO).

Double-Stranded RNA-Mediated Interference of Endogenous Rab1. A double-stranded siRNA with 19-nucleotide duplex RNA and 2-nucleotide 3' dTdT overhangs (sense, 5'-GAUCCGAACCAUCGAGCUGdTdT-3', and antisense, 3'-dTdTCUAGGCUUGGUAGCUCGAC-5') targeting the sequence at the positions 136 to 156 relative to the start code (5'-AAGATCCGAACCATCGAGCTG3') of human Rab1 (accession NM030981) and a control nonsilencing siRNA duplex (sense, 5' -UUCUCCGAACGUGUCACGUdTdT-3' and antisense, 5'-dTdTAAGAGGCUUGCACAGUGCA-3') targeting a DNA sequence AATTCTCCGAACGTGTCACGT were purchased from Qiagen (Valencia, CA). BLAST searches of the database reveals that this siRNA probe is specific for Rab1 and has no homology to other Rab proteins. HEK293 cells were plated on six-well dishes at a density of $2 \times 10^{5}$ cells/well for 12 to $16 \mathrm{~h}$ before transfection. The plasmid encoding selected receptor was mixed with siRNA and simultaneously transfected by using Lipofectamine 2000 reagent as described previously (Wu et al., 2003).

Measurement of ERK1/2 Activation. HEK293 cells were cultured on six-well dishes and transfected with $1.0 \mu \mathrm{g}$ of AT2R. Thirtysix to $48 \mathrm{~h}$ after transfection, the cells were serum-starved for at least $3 \mathrm{~h}$ and then stimulated with Ang II with or without EGF. For measurement of ERK1/2 activation by EGF, HEK293 cells were transfected with the control pcDNA vector or Rab1S25N and then stimulated with EGF at a concentration of $10 \mathrm{ng} / \mathrm{ml}$ for different times $(2-20 \mathrm{~min})$ at $37^{\circ} \mathrm{C}$. The stimulation was terminated by addition of $1 \times$ SDS-gel loading buffer (Wu et al., 2003; Dong and Wu, 2007). After solubilization of the cells, $20 \mu \mathrm{l}$ of sample was separated by $12 \%$ SDS-PAGE. Activation of ERK1/2 was determined by measuring the level of ERK1/2 phosphorylation with phosphospecific ERK1/2 antibodies. The blots were then stripped and reprobed with total ERK1/2 antibodies to confirm equal loading of the gels.

Real-Time Quantitative PCR. Total RNA was isolated from HEK293 cells transfected with Rab1 using the standard RNA extraction procedure. Real-time quantitative PCR was performed in a total volume of $20 \mu \mathrm{l}$ consisting of $2 \times$ IQ SYBR Green Super mix (Bio-Rad Laboratories, Hercules, CA; containing iTaq DNA polymerase, dNTPs with dUTP, SYBR Green I, $20 \mathrm{nM}$ fluorescein, and stabilizers) and $200 \mathrm{nM}$ primers. The forward and reverse primers for AT2R were 5-CAGCAGTCTTCCTTTTGATAATCT-3 and 5-CTTATCTGCCGGTTTGTGTGAGCA-3, respectively, and the cycling conditions were $3 \mathrm{~min}$ at $95^{\circ} \mathrm{C}$, and then 50 cycles consisting of $20 \mathrm{~s}$ at $95^{\circ} \mathrm{C}$ and $30 \mathrm{~s}$ for annealing and extension at $60^{\circ} \mathrm{C}$. The $\beta$-actin mRNA level was simultaneously measured as an internal control using the forward primer 5' -TGTGATGGTGGGAATGGGTCAGAA-3' and the re- verse primer 5'-TGTGGTGCCAGATCTTCTCCATGT-3'. The AT2 mRNA level was normalized to $\beta$-actin and expressed as -fold change.

Measurement of AT2R Ubiquitination. HEK293 cells were cultured on 100-mm dishes and transfected with $3 \mu \mathrm{g}$ of AT2R-GFP and $3 \mu \mathrm{g}$ of HA-ubiquitin together with $9 \mu \mathrm{g}$ of the pcDNA vector, Rab1, or Rab1S25N. After transfection for $36 \mathrm{~h}$, the cells were treated with MG132, a proteasome inhibitor, at a concentration of 10 $\mu \mathrm{M}$ for $12 \mathrm{~h}$. The cells were then washed with PBS and incubated with $500 \mu \mathrm{l}$ of buffer containing $50 \mathrm{mM}$ Tris, $\mathrm{pH} 7.4,150 \mathrm{mM} \mathrm{NaCl}$, $1 \%$ Nonidet P-40, $0.5 \%$ sodium deoxycholate, $0.1 \%$ EDTA, and protease inhibitors at $4^{\circ} \mathrm{C}$ for $1 \mathrm{~h}$ with gentle rotation. After centrifugation for $15 \mathrm{~min}$ at $14,000 \mathrm{~g}, 20 \mu \mathrm{l}$ of protein G PLUS-agarose beads was added into the supernatant for $1 \mathrm{~h}$ to remove unspecific binding proteins. The precleared supernatant was then incubated with $2 \mu \mathrm{g}$ of anti-GFP antibodies and rotated overnight at $4^{\circ} \mathrm{C}$ followed by incubation with $12.5 \mu \mathrm{l}$ of protein G-agarose beads for $4 \mathrm{~h}$ at $4^{\circ} \mathrm{C}$. The beads were then washed twice with buffer, and the bound proteins were eluted with $2 \times$ SDS-gel loading buffer. AT2R-GFP and HAubiquitin bound to AT2R in the immunoprecipitates were detected by immunoblotting using anti-GFP and HA antibodies, respectively.

Protein Complementation Experiments. Homo- and heterodimerization of AT1R and AT2R was measured by protein complementation experiments. GFP and its variants have a property that makes them useful for detecting bimolecular protein interactions. When these proteins are split into the $\mathrm{N}$ - and $\mathrm{C}$-terminal fragments, neither fragment is fluorescent when expressed alone in cells nor does coexpression result in the reconstitution of a fluorescent protein. When these complementary $\mathrm{N}$ - and C-terminal fragments are fused to two proteins that do associate to form a complex, these two fragments can associate and correctly fold to produce a fluorescent protein (Hu et al., 2002). HEK293 cells were cultured on six-well plates and transfected with $1 \mu \mathrm{g}$ of vectors expressing the Venus1 or Venus2 fusion proteins. Twenty-four hours after transfection, cells were harvested and washed once with PBS. The cells were then suspended in PBS containing $0.1 \%$ glucose and distributed into 96-well microplates (white OptiPlate; PerkinElmer Life and Analytical Sciences, Boston, MA). Fluorescent signals were read using a Fusion instrument (PerkinElmer Life and Analytical Sciences) with a time read of $1 \mathrm{~s}$, a photomultiplier tube voltage of 500, and a gain of 1 , using a halogen light source with an excitation filter of 485/10 $\mathrm{nm}$ and an emission filter of $535 / 25 \mathrm{~nm}$ as described previously (Dupré et al., 2007).

To visualize the subcellular localization of the complemented fluorescent proteins, HEK293 cells were harvested $24 \mathrm{~h}$ after transfection and seeded on laminin-coated coverslips for $4 \mathrm{~h}$ at $37^{\circ} \mathrm{C}$. The cells were fixed for $20 \mathrm{~min}$ in $\mathrm{PBS}, \mathrm{pH} 7.4$, containing $3 \%$ paraformaldehyde, washed three times in PBS, and incubated for $1 \mathrm{~h}$ at room temperature in PBS containing 2\% normal goat serum plus $0.2 \%$ Triton X-100. Confocal microscopy was performed with an unmodified LSM-510 system (with photomultiplier tube detection; Carl Zeiss Inc., Thornwood, NY) using a 63/1.4 oil Plan-Apochromat objective. GFP (green) was excited at $488 \mathrm{~nm}$ with an argon laser, emitting fluorescence at $510 \mathrm{~nm}$ (Dupré et al., 2006, 2007).

Immunoblotting. Western blot analysis of protein expression was carried out as described previously (Wu et al., 2003). Samples were separated by SDS-PAGE and transferred onto polyvinylidene difluoride membranes. The signal was detected using ECL Plus (PerkinElmer Life and Analytical Sciences) and a luminescent image analyzer (LAS-1000 Plus; Fuji Film, Tokyo, Japan) and quantitated using the Image Gauge, version 3.4 (Fuji Film).

Statistical Analysis. Differences were evaluated using Student's $t$ test, and $p<0.05$ was considered as statistically significant. Data are expressed as the mean \pm S.E. 


\section{Results}

Role of Rab1 GTPase in the Cell Surface and Total Expression of AT2R. To determine whether AT2R targeting to the cell surface is regulated by Rab1 GTPase, we determined the effect of expressing Rab1 and its dominantnegative mutants or Rab1 siRNA on the cell surface expression, total expression, subcellular distribution, and signaling of AT2R. In the first series of experiments, HA-AT2R was transiently expressed together with Rab1 or its dominantnegative mutants Rab1S25N and Rab1N124I, and the cell surface expression of AT2R was quantified by flow cytometry after staining with anti-HA antibodies in unpermeabilized cells. The expression of wild-type Rab1 increased the cell surface expression of AT2R by 2.3-fold, whereas Rab1S25N and Rab1N124I significantly inhibited the cell surface expression of AT2R by 82 and 65\%, respectively, compared with cells transfected with the empty pcDNA3 vector (Fig. 1A).

To determine whether Rab1 expression altered total AT2R expression, GFP-tagged AT2R was expressed with Rab1, Rab1S25N, or Rab1N124I and the overall expression of AT2R was determined by flow cytometry measuring the GFP signal. The expression of Rab1 significantly enhanced total expression of AT2R by $38 \%$, whereas Rab1S25N and Rab1N124I inhibited total AT2R expression by 69 and 44\%, respectively, compared with cells transfected with the empty pcDNA3 vector (Fig. 1B). The ratio of cell surface to total expression of AT2R was markedly increased by expression of Rab1 and decreased by expression of dominant-negative Rab1 mutants (Fig. 1C). These data indicate that AT2R transport to the cell surface uses a Rab1-dependent pathway and the expression level of endogenous Rab1 is a rate-limiting factor for AT2R targeting to the plasma membrane.

In the second series of experiments, we determined the effect of Rab1S25N and Rab1N124I on the subcellular distribution of AT2R. To exclude the possibility that the epitope tagging may produce nonspecific effects on AT2R trafficking, AT2R tagged with three different epitopes (HA or Myc at the $\mathrm{N}$ terminus and GFP at the $\mathrm{C}$ terminus) was used. AT2R tagged with these different tags robustly expressed at the cell surface (Fig. 2A), suggesting that epitope tagging did not influence the subcellular localization of the receptor. Consistent with its inhibitory effect on the cell surface expression of AT2R as quantified by flow cytometry, transient expression of Rab1S25N and Rab1N124I resulted in a marked perinuclear distribution of AT2R (Fig. 2A), consistent with well established Rab1 function in regulating ER-to-Golgi protein transport. To further prove that intracellular accumulation of AT2R was indeed caused by expression of the Rab1 mutants, we determined the colocalization of HA-tagged AT2R and GFP-tagged Rab1 mutants. HA-AT2R was arrested in the perinuclear region of the cells that were transfected with GFP-Rab1S25N or GFP-Rab1N124I (Fig. 2B). AT2R did not colocalize with either Rab1 mutant (Fig. 2B) as AT2R and the dominant-negative Rab1 mutants are presumably localized to the ER/Golgi and the cytoplasm, respectively.

Effect of Rab1 on the mRNA Level and Ubiquitination of AT2R. Our preceding data demonstrated that expression of Rab1 and its dominant-negative mutants altered the overall expression of AT2R, which is in contrast to their effect on the total expression of many other exogenously expressed GPCRs, such as AT1R, $\alpha_{2 \mathrm{~B}}$-adrenergic receptor
A

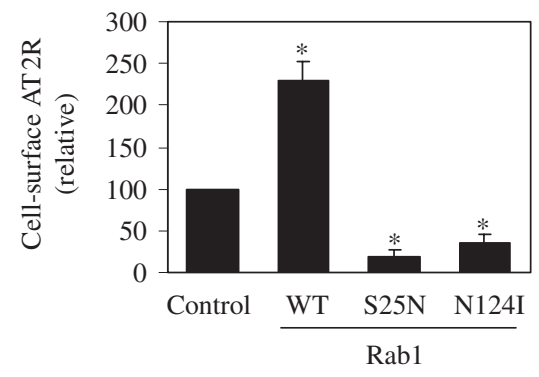

B

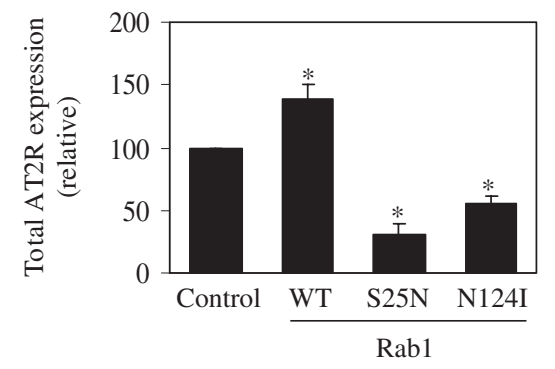

C

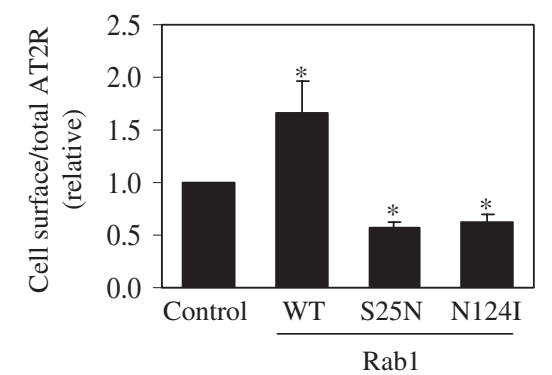

Fig. 1. Effect of Rab1 on the cell surface and total expression of AT2R. A, effect of Rab1 on AT2R expression at the cell surface. HEK293 cells were transfected with HA-AT2R together with pcDNA3.1 (Control), wild-type (WT) Rab1 or the dominant-negative Rab1 mutants Rab1S25N and Rab1N124I. AT2R expression at the cell surface was quantitated by flow cytometry after incubation with anti-HA antibodies as described under Materials and Methods. The data shown are percentage of the mean value obtained from the cells transfected with HA-AT2R and pcDNA3.1 and are presented as the mean \pm S.E. of four experiments. B, effect of Rab1 on the overall expression of AT2R. HEK293 cells were transfected with AT2R-GFP together with pcDNA3.1 (Control), WT Rab1, Rab1S25N, or Rab1N124I. Total AT2R expression was quantitated by flow cytometry measuring GFP fluorescence. The data shown are percentage of the mean value obtained from the cells transfected with AT2RGFP and pcDNA3.1 and presented as the mean \pm S.E. of four experiments. C, ratio of cell surface AT2R to total AT2R expression generated from the data shown in A and B. The data presented as the mean \pm S.E. of four experiments. *, $P<0.05$ versus control.

(AR), and $\beta_{2}$-AR in HEK293 cells (Wu et al., 2003). Therefore, it is interesting to further study how Rab1 influences total AT2R expression. We first determined whether Rab1 expression could alter total mRNA level of AT2R. HEK293 cells were transiently transfected with AT2R and Rab1 or Rab1S25N and the total mRNA level of AT2R was determined by reverse transcription-PCR using $\beta$-actin as a reference. Surprisingly, expression of Rab1 markedly increased total AT2R mRNA by 79\%, whereas expression of Rab1S25N only slightly, but not significantly, reduced total AT2R mRNA by $12 \%$ (Fig. 3A). These data indicate that transcriptional regulation of Rab1 contributes to the increased total AT2R expression but not reduced AT2R expression.

We then determined whether inhibition of total AT2R synthesis caused by expression of Rab1S25N was due to enhanced proteolysis of the receptor. It has been demonstrated that 
A
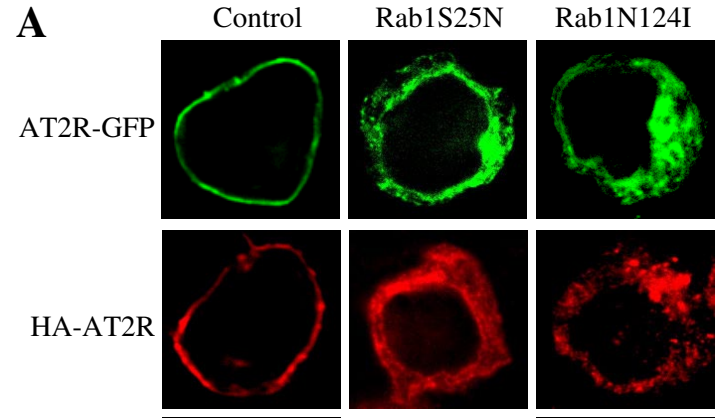

Myc-AT2R
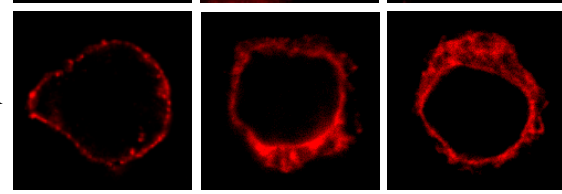

B
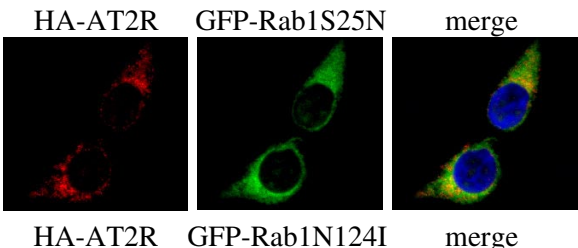

GFP-Rab1N124I
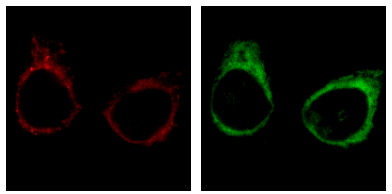

merge

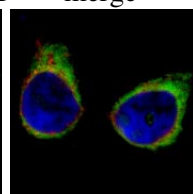

Fig. 2. Effect of Rab1 on the subcellular distribution of AT2R. A, HEK293 cells cultured on coverslips were transfected with AT2R-GFP (top), 3HAAT2R (middle), or Myc-AT2R (bottom) together with pcDNA3.1 (Control), Rab1S25N, or Rab1N124I. Subcellular distribution of AT2R-GFP was revealed by detecting GFP fluorescence and 3HA-AT2R and Myc-AT2R by fluorescence microscopy after immunostaining with rhodamine-conjugated anti-HA and anti-Myc antibodies (1:50), respectively, as described under Materials and Methods. B, colocalization of AT2R and Rab1. HEK293 cells were transfected with HA-AT2R and GFP-Rab1, and their subcellular distribution and colocalization were visualized by fluorescence microscopy after staining with rhodamine-conjugated anti-HA antibodies. The data shown are representative images from at least three separate experiments. Scale bars, $10 \mu \mathrm{m}$.

GPCR degradation may be mediated through ubiquitinationdependent pathway. Therefore, we determined whether expression of Rab1S25N could promote ubiquitination of AT2R. GFPtagged AT2R and HA-tagged ubiquitin were coexpressed in HEK293 cells, and the ubiquitination state of AT2R was determined by immunoblotting for the HA-tagged ubiquitin after immunoprecipitation of the receptor using anti-GFP antibodies. In the absence of MG132, a proteasome inhibitor, AT2R in the immunoprecipitates was not detectable. In the presence of MG132 to inhibit protein degradation, AT2R was found in the anti-GFP immunoprecipitates and the amount of AT2R-GFP in the immunoprecipitates from cells expressing Rab1 and Rab1S25N was much greater than that from control cells (Fig. $3 \mathrm{~B})$, suggesting that expression of Rab1 and RabS25N indeed promote AT2R degradation. Likewise, the amount of HA-ubiquitin bound to AT2R from cells expressing Rab1 and Rab1S25N was much higher than that from control cells. These data indicate that AT2R undergoes ubiquitination that is modulated by expression of Rab1.

Inhibition of the Cell Surface Expression and Intracellular Accumulation of AT2R by siRNA-Mediated Knockdown of Rab1. To further define the function of Rab1
A

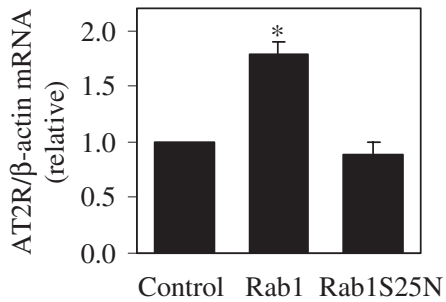

B

IP: anti-GFP

$\begin{array}{rcccc}\text { AT2R-GFP } & + & + & + & + \\ \text { HA-ubiquitin } & + & + & + & + \\ \text { pcDNA } & + & + & - & - \\ \text { Rab1 } & - & - & + & - \\ \text { Rab1S25N } & - & - & - & + \\ \text { MG132 } & - & + & + & +\end{array}$

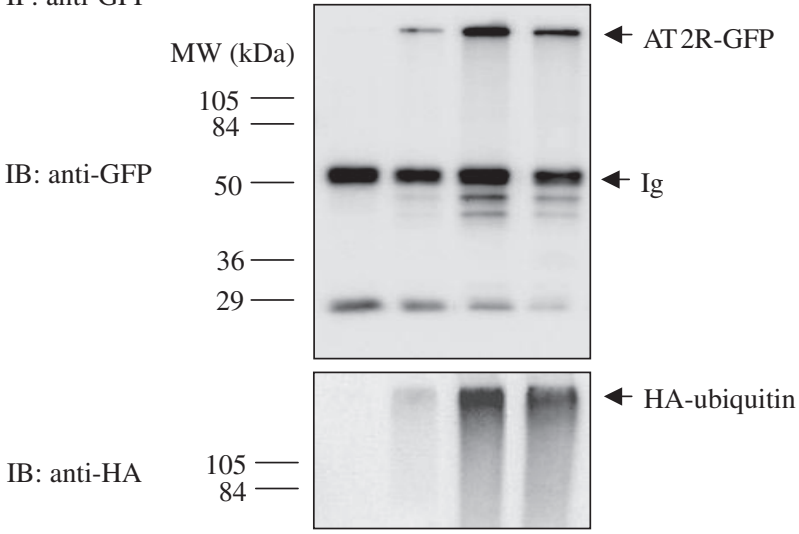

Fig. 3. Effect of Rab1 on the mRNA levels and ubiquitination of AT2R. A effect of Rab1 and Rab1S25N on the mRNA levels of AT2R. HEK293 cells were transfected with AT2R together with pcDNA3.1 (Control), wild-type Rab1, or Rab1S25N. The mRNA levels of AT2R and $\beta$-actin were measured by reverse transcription-PCR. The data shown are the mean value relative to cells transfected with HA-AT2R and pcDNA3.1 and are presented as the mean \pm S.E. of three experiments each in triplicate. $*, P<$ 0.05 versus control. $B$, effect of Rab1 on the ubiquitination state of AT2R. HEK293 cells were transfected with AT2R-GFP and HA-ubiquitin plus pcDNA3.1, Rab1, or Rab1S25N and treated with MG132 as described under Materials and Methods. After immunoprecipitation with anti-GFP antibodies, AT2R-GFP and HA-ubiquitin were detected by immunoblotting using anti-GFP (top) and anti-HA (bottom) antibodies, respectively. Similar results were obtained in two separate experiments. Molecular mass is indicated in the left.

in regulating AT2R trafficking, we determined the effect of siRNA-mediated knockdown of endogenous Rab1 on the cell surface expression and subcellular distribution of AT2R. Introduction of Rab1 siRNA into HEK293 cells dramatically reduced the Rab1 expression level compared with control cells without transfection or transfected with control siRNA (Fig. 4A). In contrast, Rab1 siRNA transfection had no effect on the expression of the closely related small GTPases Rab2, Rab4, and Rab6 (Fig. 4A), demonstrating that the Rab1 siRNA selectively altered Rab1 expression. Similar to the dominant-negative Rab1 mutants, siRNA-mediated knockdown of endogenous Rab1 also induced a decrease in the cell surface expression of AT2R (Fig. 4B). Consistently, subcellular localization showed that AT2R was accumulated in the perinuclear regions of transfected cells and was unable to reach the cell surface (Fig. $4 \mathrm{C}$ ). These data strongly demonstrated that similar to AT1R (Wu et al., 2003; Filipeanu et al., 2004), AT2R transport to the cell surface depends on the normal function of Rab1 GTPase.

Effect of Attenuation of Rab1 Function on AT2R Signaling. It has been demonstrated that AT2R inhibits EGF- 


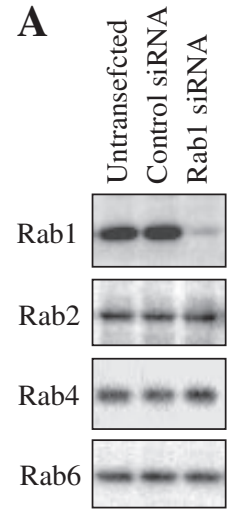

- Control siRNA $\square$ Rab1 siRNA

B

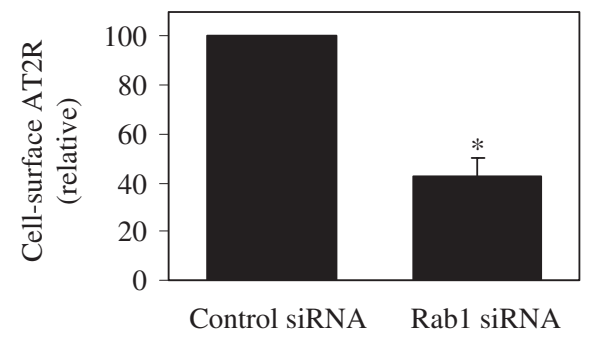

C

Control siRNA
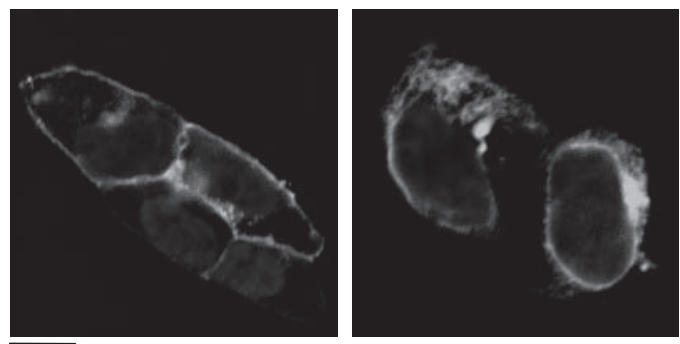

Fig. 4. Effect of siRNA-mediated knockdown of Rab1 on the cell surface expression and subcellular distribution of AT2R. A, HEK293 cells cultured in six-well dishes were transiently transfected with control siRNA or Rab1 siRNA as described under Materials and Methods. At $48 \mathrm{~h}$ after transfection, total homogenate protein was separated by $12 \%$ SDS-PAGE, and the expression levels of Rab1, Rab2, Rab4, and Rab6 were determined by immunoblotting using Rab isoform-specific antibodies (left). Quantitative data are expressed as percentages of Rab protein expression in cells transfected with control siRNA and are presented as the means \pm S.E. of three individual experiments (right). $*, P<0.05$ versus control siRNA. B, effect of Rab1 siRNA on AT2R expression at the cell surface. HEK293 cells were transfected with HA-AT2R together with control or Rab1 siRNA as described under Materials and Methods. AT2R expression at the cell surface was measured by flow cytometry after staining by anti-HA antibodies. The data shown are percentage of the mean value obtained from the cells transfected with HA-AT2R and control siRNA and are presented as the mean \pm S.E. of three experiments. $*, P<0.05$ versus control siRNA. C, effect of Rab1 siRNA on AT2R subcellular localization. HEK293 cells were transfected with GFP-conjugated AT2R together with control or Rab1 siRNA as described above. The subcellular distribution of AT2R was revealed by detecting GFP fluorescence. The data are representative images of at least four individual experiments. Scale bar, $10 \mu \mathrm{m}$.

mediated ERK1/2 activation (De Paolis et al., 2002). To determine whether Rab1 is capable of regulating AT2R signaling through modifying receptor export to the cell surface, we determined whether transient expression of Rab1S25N could alter the inhibitory effect of AT2R on EGFmediated ERK1/2 activation. AT2R was transiently expressed with or without expression of Rab1S25N in HEK293 cells and stimulated with EGF alone or EGF plus Ang II.
EGF stimulation potently activated ERK1/2, which can be inhibited by Ang II stimulation in cells expressing AT2R. Attenuation of EGF-mediated ERK1/2 activation by AT2R was markedly inhibited in cells expressing Rab1S25N (Fig. $5 \mathrm{~A})$. These data suggest that Rab1S25N blocks AT2R transport to the cell surface and attenuates its function.

To determine whether the effect of Rab1S25N on ERK1/2 activation by EGF and Ang II/AT2R was due to direct influence of Rab1S25N on the EGF-mediated ERK1/2 activation, we measured the effect of Rab1S25N on EGF-mediated ERK1/2 activation. EGF stimulation robustly activated ERK1/2 in a time- and dose-dependent manner (Fig. 5, B and $5 \mathrm{C}$ ), and ERK1/2 activation by EGF was almost identical in cells transfected with the control empty vector compared with cells transfected with Rab1S25N. These data suggest that inhibition of Rab1 function does not significantly influence EGF-mediated ERK1/2 activation and imply that Rab1 modulation of AT2R effect on EGF-mediated ERK1/2 activation is not due to the effect of Rab1 on the EGF/EGFRmediated ERK1/2 activation per se.

A
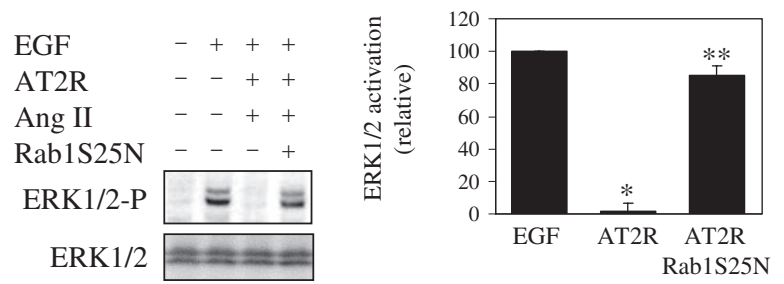

B
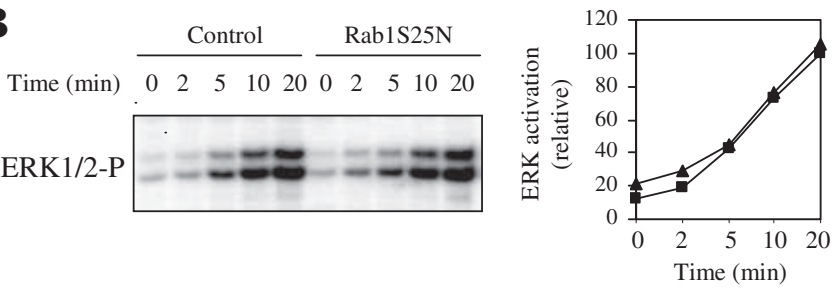

C
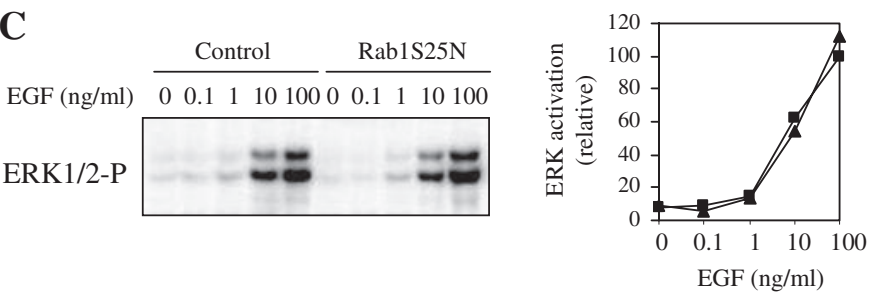

Fig. 5. Effect of Rab1 on AT2R function in regulating EGF-mediated ERK1/2 activation. A, attenuation of AT2R inhibition on EGF-activated ERK1/2 by Rab1S25N. HEK293 cells cultured on six-well dishes were transfected with AT2R-GFP with or without Rab1S25N and then stimulated with EGF (100 ng/ml) alone or EGF plus Ang II (1 $\mu \mathrm{M})$ for $10 \mathrm{~min}$ at $37^{\circ} \mathrm{C}$. ERK1/2 activation was determined by Western blot analysis using phosphospecific ERK1/2 antibodies. Left, representative blots of ERK1/2 activation (top) and total ERK2 expression (bottom); right, quantitative data are expressed as percentages of ERK1/2 activation obtained from cells transfected with the pcDNA3 vector and stimulated with EGF and are presented as the mean \pm S.E. of three experiments. $*, P<0.05$ versus cells stimulated with EGF alone; **, $P<0.05$ versus cells transfected with AT2R and stimulated with EGF and Ang II. B and C, effect of Rab1 on EGF-mediated ERK1/2 activation. HEK293 cells cultured on six-well dishes were stimulated with EGF at a concentration of $10 \mathrm{ng} / \mathrm{ml}$ for different times (2-20 $\mathrm{min}$ ) (B) or with increasing concentrations of EGF $(0.1-100 \mathrm{ng} / \mathrm{ml})$ for $10 \mathrm{~min}(\mathrm{C})$ at $37^{\circ} \mathrm{C}$. Left, representative blots of ERK1/2 activation. There is no significant difference in total ERK1/2 expression (data not shown). Right, quantitative data expressed as percentages of ERK1/2 activation obtained from cells stimulated with EGF for $20 \mathrm{~min}$ (B) and with EGF at a concentration of $100 \mathrm{ng} / \mathrm{ml}$ (C). Similar results were obtained in three independent experiments. 
Dimerization of the ER-Retained AT1R Mutant with Wild-Type AT1R and AT2R. AT1R and AT2R have been well demonstrated to form homodimers and heterodimers (AbdAlla et al., 2004; Hansen et al., 2004; Miura et al., 2006). We have recently demonstrated that the AT1Rm, in which the $\mathrm{F}(\mathrm{X})_{6} \mathrm{LL}$ motif in the membrane-proximal $\mathrm{C}$ terminus was mutated, was unable to transport to the cell surface and was arrested in the ER (Duvernay et al., 2004). We first asked whether the export-deficient AT1Rm retained its ability to form homodimers as measured using a protein complementation assay based on the reconstitution of the GFP variant Venus. As shown in Fig. 6A, both wild-type AT1R and AT2R formed homodimers, reconstituting Venus fluorescence compared with negative controls consistent with many
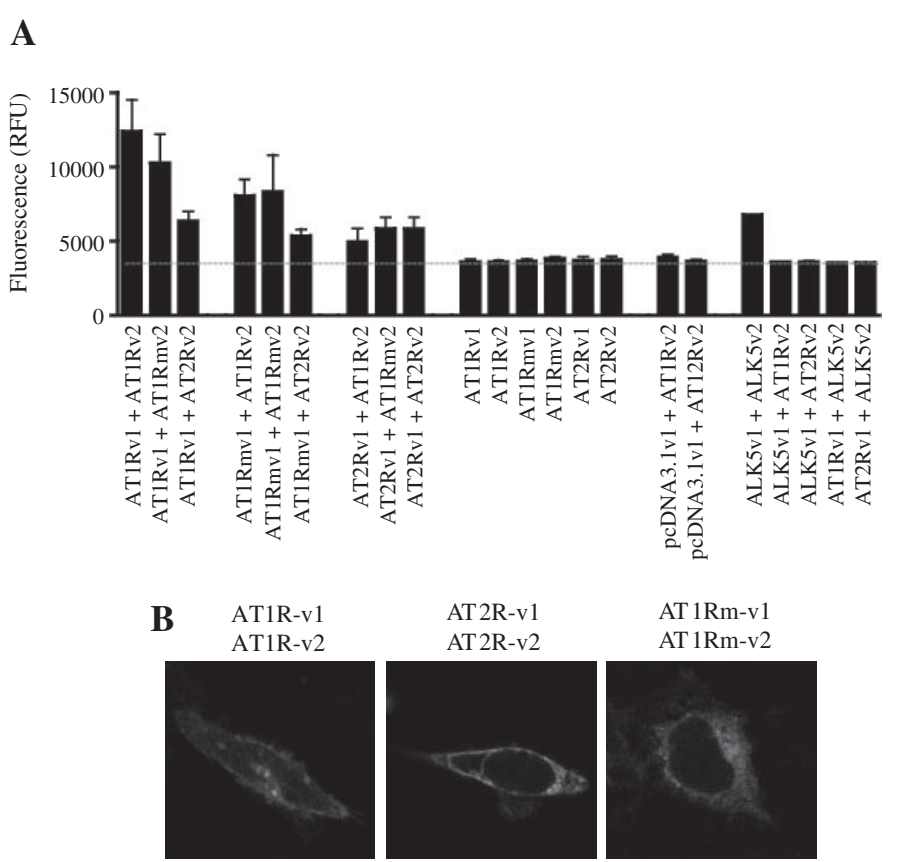

$\mathbf{C}$ HA-AT2R localization

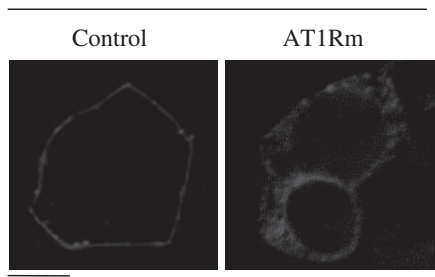

Fig. 6. Protein complementation assay demonstrating dimerization of AT1R, AT2R, and AT1Rm. A, reconstitution of Venus GFP fluorescence when split pairs were used but not when individual splits were expressed alone. Homodimerization of ALK5 was used as a positive control and dimerization between ALK5 and AT1R or AT2R as negative controls. Position of Venus1 or Venus2 did not affect the fluorescent signal generated. The data are presented as the mean \pm S.E. of three experiments. B, confocal microscopy showing reconstitution of Venus GFP at the cell surface as well as intracellular compartments when split AT1R/AT1R and AT2R/AT2R homodimers were coexpressed while mutant AT1R dimers were retained inside the cell. Data are representative of three independent experiments. C, subcellular distribution of AT2R in the absence and presence of AT1Rm. HEK293T cells cultured on coverslips were transfected with HA-AT2R plus the pEGFP-N1 vector (Control) or GFP-tagged AT1Rm. Subcellular distribution of HA-AT2R was revealed by fluorescence microscopy after staining with anti-HA antibodies in permeabilized cells as described under Materials and Methods. The data are representative images from at least three independent experiments. Scale bar, $10 \mu \mathrm{m}$. reports in the literatures (AbdAlla et al., 2004; Hansen et al., 2004; Miura et al., 2006). Similar to wild-type AT1R and AT2R, the mutant AT1Rm also formed homodimers (Fig. 6A). Under the same experimental conditions, the activin-like kinase (ALK) 5 (a transforming growth factor- $\beta$ receptor) formed homodimers but did not form heterodimers with either AT1R or AT2R (Fig. 6A). We then used confocal microscopy to visualize the subcellular localization of reconstituted dimers that would indicate where AT1R, AT2R, and AT1Rm formed homodimers. As shown in Fig. 6B, the GFP fluorescence was observed both at the cell surface and intracellular compartments in cells expressing either AT1R or AT2R, suggesting that AT1R and AT2R could form dimers at the plasma membrane and intracellular compartments. In contrast, the GFP fluorescence was seen only in intracellular compartments, presumably ER, but not at the plasma membrane, in cells expressing AT1Rm (Fig. 6B).

We next determined whether AT1Rm could undergo heterodimerization with wild-type AT1R and AT2R. Similar to heterodimerization between wild-type AT1R and AT2R, protein complementation experiments again showed that AT1Rm heterodimerized with both AT1R and AT2R (Fig. $6 \mathrm{~A})$. These data demonstrate that mutation of the $\mathrm{F}(\mathrm{X})_{6} \mathrm{LL}$ motif in the $\mathrm{C}$ termini does not prevent receptor dimerization. It is important to note that these data suggest that AT1R and AT2R dimerization (homo- and heterodimerization) occurs constitutively during their biosynthesis and maturation in the ER.

To determine whether the ER-retained AT1Rm could function as a dominant-negative mutant inhibiting AT2R transport to the cell surface, AT1Rm was expressed together with HA-AT2R in HEK293 cells, and the cell surface expression and subcellular distribution of AT2R were analyzed. The subcellular localization of the receptors showed that AT2R was trapped in the perinuclear region, presumably in the ER (Fig. 6B). These data indicate that AT1Rm may exert a dominant-negative effect on AT2R cell surface targeting.

\section{Discussion}

The physiological function of Ang II is mediated through activating AT1R and AT2R at the plasma membrane, which couple to different $\mathrm{G}$ proteins to produce largely opposite effects. We have characterized previously the regulation of exit from the ER and subsequent transport from the ER through the Golgi to the cell surface of AT1R (Wu et al., 2003; Duvernay et al., 2004; Filipeanu et al., 2004; Dong and Wu, 2007; Dong et al., 2008). However, the regulators involved in the AT2R export trafficking and the role of export trafficking in AT2R signaling remain poorly defined. Here, we have demonstrated that the small GTPase Rab1 is an important determinant of surface trafficking AT2R. We have also shown that both homo- and heterodimerization of AT1R and AT2R occur in the ER and that a single defective trafficking signal in one monomer equivalent in a receptor dimer is sufficient to block cell surface transport, suggestive of an important quality control step in the function of dimeric GPCRs.

Rab proteins represent the largest branch of the Ras-like small GTPase superfamily. Rab1 GTPase specifically modulates ER-to-Golgi protein transport (Plutner et al., 1991; Jin et al., 1996; Lupashin and Waters, 1997; Allan et al., 2000). 
As an initial approach to elucidating the molecular mechanism of GPCR export trafficking, we have determined the role of Rab1 in the cell surface targeting of several GPCRs, including AT1R, $\alpha_{1}-\mathrm{AR}, \alpha_{2 \mathrm{~B}}-\mathrm{AR}, \beta_{1}-\mathrm{AR}$, and $\beta_{2}$-AR (Wu et al., 2003; Filipeanu et al., 2004, 2006). Our previous studies have shown that the ER-to-cell surface transport of different GPCRs is mediated through distinct pathways. Whereas $\alpha_{2 \mathrm{~B}}$-AR transport is independent of Rab1, AT1R export is dependent on Rab1 (Wu et al., 2003; Filipeanu et al., 2004, 2006). Interestingly, augmentation of Rab1 function by overexpressing wild-type Rab1 facilitates the cell surface transport of endogenous AT1R in cardiomyocytes but not exogenously expressed AT1R in HEK293 cells (Wu et al., 2003; Filipeanu et al., 2004). Based on these sensitivities, ER-tocell surface transport of GPCRs can be divided into three different pathways (Dong et al., 2007). The first pathway is inhibited by attenuating Rab1 function and facilitated by increasing Rab1 function and is used by AT1R and $\alpha_{1}$-AR in cardiac myocytes. The second pathway is inhibited by attenuating Rab1 function, but not sensitive to increasing Rab1 function (such as $\beta_{1}-\mathrm{AR}$ and $\beta_{2}-\mathrm{AR}$ ). The third pathway is not influenced by manipulating Rab1 function and is used by $\alpha_{2 \mathrm{~B}}-\mathrm{AR}$. Our current data indicate that AT2R cell surface transport is inhibited by the dominant-negative Rab1 mutants and Rab1 siRNA and facilitated by overexpressing Rab1, indicating that AT2R export is mediated through a Rab1-dependent pathway and the endogenous Rab1 level is a rate-limiting factor for its transport.

It is apparent that AT1R and AT2R have distinct sensitivities to the manipulation of Rab1 function. First, attenuating Rab1 function inhibits more potently the cell surface expression of AT2R than AT1R. Our previous and current data demonstrate that Rab1S25N inhibits the cell surface expression of AT1R and AT2R by 65 and $82 \%$, respectively, in HEK293 cells (Wu et al., 2003). Likewise, Rab1N124I inhibits the cell surface expression of AT1R and AT2R by 45 and $65 \%$, respectively (Wu et al., 2003). Such a moderate inhibition of Rab1N124I on endogenous AT1R was also observed in neonatal cardiomyocytes by $36 \%$ and in HL-1 myocytes by 44\% (Filipeanu et al., 2004). Second, responses of AT1R and AT2R to the augmentation of Rab1 function are also different. Our previous data demonstrated that the cell surface transport of exogenously expressed AT1R was not altered by overexpressing Rab1 in HEK293 cells (Wu et al., 2003). In contrast, Rab1 overexpression increased AT2R at the cell surface by $130 \%$ in HEK293 cells. These data indicate that functional manipulation of components of the transport machinery such as Rab1 to alter protein ER-to-Golgi transport along the secretory pathway may provide a novel means for differential modulation of the export trafficking of AT1R and AT2R.

The opposing effects of Rab1 and Rab1S25N on the total AT2R synthesis are likely mediated through distinct molecular mechanisms. Similar to cell surface expression, total AT2R is enhanced by Rab1 and attenuated by Rab1 mutants. These data are in contrast to our previous data showing that Rab1 did not significantly alter the overall expression of several exogenously expressed GPCRs, including AT1R (Wu et al., 2003). We have further demonstrated that Rab1 expression markedly increased the AT2R mRNA level, suggesting that Rab1-enhanced cell surface expression of AT2R is at least in part due to increased AT2R synthesis. These data also suggest that Rab1, as a well characterized trafficking regulator, is able to modulate protein synthesis at the transcriptional level. However, the molecular mechanism underlying Rab1 function in modulating AT2R synthesis needs further investigation. It is possible that Rab1 overexpression may alter mRNA stability, which would eventually influence protein synthesis. Consistent with this possibility, a recent study has demonstrated that Rab1 depletion significantly decreases the RNA levels of hepatitis $\mathrm{C}$ virus (Sklan et al., 2007). However, Rab1S25N had no effect on the mRNA levels of AT2R. Rather, it dramatically enhanced AT2R ubiquitination. These data indicate that similar to many other GPCRs, AT2R undergoes ubiquitination and ubiquitinationdependent proteolysis seems to be main determinant for the reduction in the total AT2R expression caused by Rab1S25N. Interestingly, although expression of Rab1 and Rab1S25N results in opposing effects on the total and cell surface expression of AT2R, they similarly enhance AT2R ubiquitination. It is possible that the Rab1-promoted AT2R ubiquitination is caused by the increase in the total AT2R synthesis induced by Rab1 expression. Rab1 overexpression markedly facilitates total AT2R synthesis, which will contribute to the increased cell surface expression of the receptor. Meanwhile, not all the newly synthesized AT2R are able to export from the ER and transport to the cell surface, some receptors will remain in the ER and then target to the degradation pathway via ubiquitination.

It is important to note that our previous results and the current study strongly indicate that, in addition to modulating export trafficking of AT1R and AT2R, Rab1 is also capable of regulating their function. It has been well demonstrated that AT1R and AT2R couple to different G proteins and activate opposite signal transduction pathways (Murphy et al., 1991; Mehta and Griendling, 2007). We have shown previously that increase Rab1 expression facilitates AT1R function in regulating ERK1/2 activation and growth in cardiac myocytes (Wu et al., 2001; Filipeanu et al., 2004). Here, we demonstrated that expression of dominant-negative Rab1 mutants also inhibited AT2R function. Rab1 function in regulating AT1R and AT2R signaling is probably mediated through modulating their ER-to-Golgi trafficking, which would eventually alter the number of the receptors at the cell surface. Therefore, our studies provide a possibility to manipulate signaling of very closely related GPCRs (e.g., AT1R versus AT2R) by manipulating their trafficking along the early secretory pathway.

AT1R and AT2R form homodimers and heterodimers (AbdAlla et al., 2004; Hansen et al., 2004; Miura et al., 2006). However, where they form dimers and the role of the dimerization in the ER export remain elusive. Our current data have demonstrated that AT1R and AT2R dimers could be detected in both intracellular compartments and at the plasma membrane. Furthermore, similar to AT1R, the AT1R mutant defective in the ER export homodimerized and heterodimerized with AT1R and AT2R. These data indicate that homo- and heterodimerization of AT1R and AT2R occur constitutively and as early as in the ER. More interesting to note is that the AT1R mutant markedly blocked the cell surface transport of wild-type AT2R revealed by subcellular distribution. These data suggest that AT1R mutant may function as a dominant-negative mutant to block the transport of wild-type AT2R. The inhibition of AT2R cell surface expression by the AT1R mutant is probably mediated through heterodimerization between two receptors. The AT1R mutant defective in the ER export forms heterodimers with wild-type AT2R and traps AT2R inside the cell. This pos- 
sibility is strongly supported by the inhibition of cell surface expression of GPCRs by heterodimerization of wild-type receptors with their intracellularly retained receptor mutants. These data provide strong evidence implicating that homo- and heterodimerization of AT1R and AT2R play an essential role in their export from the ER and subsequent transport to the cell surface. These data also provide a useful means for manipulating the function of Ang II receptors by using their exportdefective mutants.

In summary, our previous and current studies have demonstrated that the cell surface and total expression of AT1R and AT2R have different sensitivities to the manipulation of Rab1 function, although export trafficking of both receptors is mediated through a Rab1-dependent pathway. The ER export-defective AT1R mutant functions as a dominant-negative mutant inhibiting AT2R transport to the cell surface, which is mediated through heterodimerization, suggesting that AT1R and AT2R constitutively form dimers in the ER and that dimerization plays an important role in their export from the ER. Taken together, these data provide strong evidence indicating that the export trafficking of AT1R and AT2R is mediated through distinct, highly regulated processes and that the function of Ang II may be manipulated through modulating its receptor anterograde trafficking along the early secretory pathway.

\section{Acknowledgments}

We are grateful to Dr. Kenneth E. Bernstein, Stephen Michnick, and Ashok K. Pullikuth for sharing reagents. We also thank Carlis Rejon for generating the ALK5 constructs and Connie Porretta and Dr. Ping Zhang (Department of Medicine, Louisiana State University Health Sciences Center, New Orleans, LA) for measuring receptor expression by flow cytometry.

\section{References}

AbdAlla S, Lother H, Langer A, el Faramawy Y, and Quitterer U (2004) Factor XIIIA transglutaminase crosslinks AT1 receptor dimers of monocytes at the onset of atherosclerosis. Cell 119:343-354.

Allan BB, Moyer BD, and Balch WE (2000) Rab1 recruitment of p115 into a cisSNARE complex: programming budding COPII vesicles for fusion. Science 289: 444-448.

Altan-Bonnet N, Sougrat R, Liu W, Snapp EL, Ward T, and Lippincott-Schwartz J (2006) Golgi inheritance in mammalian cells is mediated through endoplasmic reticulum export activities. Mol Biol Cell 17:990-1005.

Ballabeni A, Melixetian M, Zamponi R, Masiero L, Marinoni F, and Helin K (2004) Human geminin promotes pre-RC formation and DNA replication by stabilizing CDT1 in mitosis. EMBO J 23:3122-3132

De Paolis P, Porcellini A, Savoia C, Lombardi A, Gigante B, Frati G, Rubattu S, Musumeci B, and Volpe M (2002) Functional cross-talk between angiotensin II and epidermal growth factor receptors in NIH3T3 fibroblasts. J Hypertens 20:693-699.

Dong C, Filipeanu CM, Duvernay MT, and Wu G (2007) Regulation of G proteincoupled receptor export trafficking. Biochim Biophys Acta 1768:853-870.

Dong C and Wu G (2007) Regulation of anterograde transport of adrenergic and angiotensin II receptors by Rab2 and Rab6 GTPases. Cell Signal 19:2388-2399.

Dong C, Zhou F, Fugetta EK, Filipeanu CM, and Wu G (2008) Endoplasmic reticulum export of adrenergic and angiotensin II receptors is differentially regulated by Sar1 GTPase. Cell Signal 20:1035-1043.

Dupré DJ, Baragli A, Rebois RV, Ethier N, and Hébert TE (2007) Signalling complexes associated with adenylyl cyclase II are assembled during their biosynthesis. Cell Signal 19:481-489.

Dupré DJ, Robitaille M, Ethier N, Villeneuve LR, Mamarbachi AM, and Hébert TE (2006) Seven transmembrane receptor core signaling complexes are assembled prior to plasma membrane trafficking. J Biol Chem 281:34561-34573.

Duvernay MT, Dong C, Zhang X, Robitaille M, Hebert TE and Wu G (2009) A single conserved leucine residue on the first intracellular loop regulates ER export of $G$ Protein-coupled receptors. Traffic 10:552-566.

Duvernay MT, Zhou F, and Wu G (2004) A conserved motif for the transport of G protein-coupled receptors from the endoplasmic reticulum to the cell surface. $J$ Biol Chem 279:30741-30750.

Feng YH, Sun Y, and Douglas JG (2002) Gbeta gamma-independent constitutive association of Galpha s with SHP-1 and angiotensin II receptor AT2 is essential in AT2-mediated ITIM-independent activation of SHP-1. Proc Natl Acad Sci U S A 99:12049-12054.
Filipeanu CM, Zhou F, Claycomb WC, and Wu G (2004) Regulation of the cell surface expression and function of angiotensin II type 1 receptor by Rab1-mediated endoplasmic reticulum-to-Golgi transport in cardiac myocytes. J Biol Chem 279:4107741084

Filipeanu CM, Zhou F, Lam ML, Kerut KE, Claycomb WC, and Wu G (2006 Enhancement of the recycling and activation of beta-adrenergic receptor by Rab4 GTPase in cardiac myocytes. J Biol Chem 281:11097-11103.

Gallinat S, Busche S, Raizada MK, and Sumners C (2000) The angiotensin II type 2 receptor: an enigma with multiple variations. Am J Physiol Endocrinol Metab 278:E357-E374.

Hansen JL, Theilade J, Hauns $\varnothing$ S, and Sheikh SP (2004) Oligomerization of wild type and nonfunctional mutant angiotensin II type I receptors inhibits G $\alpha$ q protein signaling but not ERK activation. J Biol Chem 279:24108-24115.

Hein L, Barsh GS, Pratt RE, Dzau VJ, and Kobilka BK (1995) Behavioural and cardiovascular effects of disrupting the angiotensin II type-2 receptor in mice. Nature 377:744-747.

Hu CD, Chinenov Y, and Kerppola TK (2002) Visualization of interactions among bZIP and Rel family proteins in living cells using bimolecular fluorescence complementation. Mol Cell 9:789-798.

Huang XC, Richards EM, and Sumners C (1996) Mitogen-activated protein kinases in rat brain neuronal cultures are activated by angiotensin II type 1 receptors and inhibited by angiotensin II type 2 receptors. J Biol Chem 271:15635-15641.

Hunyady L and Catt KJ (2006) Pleiotropic AT1 receptor signaling pathways medi ating physiological and pathogenic actions of angiotensin II. Mol Endocrinol 20: 953-970.

Jin M, Saucan L, Farquhar MG, and Palade GE (1996) Rab1a and multiple other Rab proteins are associated with the transcytotic pathway in rat liver. $J$ Biol Chem 271:30105-30113

Jones KA, Borowsky B, Tamm JA, Craig DA, Durkin MM, Dai M, Yao WJ, Johnson M, Gunwaldsen C, Huang LY, et al. (1998) GABA(B) receptors function as a heteromeric assembly of the subunits GABA(B)R1 and GABA(B)R2. Nature 396: $674-679$.

Kang J, Posner P, and Sumners C (1994) Angiotensin II type 2 receptor stimulation of neuronal $\mathrm{K}+$ currents involves an inhibitory GTP binding protein. Am J Physiol 267:C1389-C1397.

Lupashin VV and Waters MG (1997) t-SNARE activation through transient inter action with a rab-like guanosine triphosphatase. Science 276:1255-1258.

Matsubara H (1998) Pathophysiological role of angiotensin II type 2 receptor in cardiovascular and renal diseases. Circ Res 83:1182-1191.

Mehta PK and Griendling KK (2007) Angiotensin II cell signaling: physiological and pathological effects in the cardiovascular system. Am J Physiol Cell Physiol 292 C82-C97.

Miura S, Fujino M, Hanzawa H, Kiya Y, Imaizumi S, Matsuo Y, Tomita S, Uehara Y, Karnik SS, Yanagisawa H, et al. (2006) Molecular mechanism underlying inverse agonist of angiotensin II type 1 receptor. J Biol Chem 281:19288-19295.

Murphy TJ, Alexander RW, Griendling KK, Runge MS, and Bernstein KE (1991) Isolation of a cDNA encoding the vascular type-1 angiotensin II receptor. Nature 351:233-236.

Overton MC and Blumer KJ (2000) G-protein-coupled receptors function as oligomers in vivo. Curr Biol 10:341-344.

Overton MC and Blumer KJ (2002) The extracellular N-terminal domain and transmembrane domains 1 and 2 mediate oligomerization of a yeast $\mathrm{G}$ protein-coupled receptor. J Biol Chem 277:41463-41472.

Plutner H, Cox AD, Pind S, Khosravi-Far R, Bourne JR, Schwaninger R, Der CJ, and Balch WE (1991) Rab1b regulates vesicular transport between the endoplasmic reticulum and successive Golgi compartments. J Cell Biol 115:31-43.

Sato K and Nakano A (2004) Reconstitution of coat protein complex II (COPII) vesicle formation from cargo-reconstituted proteoliposomes reveals the potential role of GTP hydrolysis by Sar1p in protein sorting. J Biol Chem 279:1330-1335.

Sklan EH, Serrano RL, Einav S, Pfeffer SR, Lambright DG, and Glenn JS (2007) TBC1D20 is a Rab1 GTPase-activating protein that mediates hepatitis $\mathrm{C}$ virus replication. J Biol Chem 282:36354-36361.

Stephens DJ and Pepperkok R (2004) Differential effects of a GTP-restricted mutant of Sar1p on segregation of cargo during export from the endoplasmic reticulum. J Cell Sci 117:3635-3644.

Szidonya L, Cserzo M, and Hunyady L (2008) Dimerization and oligomerization of G-protein-coupled receptors: debated structures with established and emerging functions. J Endocrinol 196:435-453.

Wu G, Yussman MG, Barrett TJ, Hahn HS, Osinska H, Hilliard GM, Wang X, Toyokawa T, Yatani A, Lynch RA, et al. (2001) Increased myocardial Rab GTPase expression: a consequence and cause of cardiomyopathy. Circ Res 89:1130-1137.

Wu G, Zhao G, and He Y (2003) Distinct pathways for the trafficking of angiotensin II and adrenergic receptors from the endoplasmic reticulum to the cell surface: Rab1-independent transport of a G protein-coupled receptor. J Biol Chem 278: 47062-47069.

Zhang J and Pratt RE (1996) The AT2 receptor selectively associates with Gialpha2 and Gialpha3 in the rat fetus. J Biol Chem 271:15026-15033.

Zhou F, Filipeanu CM, Duvernay MT, and Wu G (2006) Cell-surface targeting of alpha2-adrenergic receptors - inhibition by a transport deficient mutant through dimerization. Cell Signal 18:318-327.

Address correspondence to: Dr. Guangyu Wu, Department of Pharmacology and Experimental Therapeutics, Louisiana State University Health Sciences Center, 1901 Perdido St., New Orleans, LA 70112. E-mail: gwu@lsuhsc.edu 